\title{
Enhanced Health Facility Surveys to Support Malaria Control and Elimination across Different Transmission Settings in the Philippines
}

\author{
Ralph A. Reyes, ${ }^{1 \star}$ Kimberly M. Fornace, ${ }^{2}$ Maria Lourdes M. Macalinao, ${ }^{1}$ Beaulah L. Boncayao, ${ }^{1}$ Ellaine S. De La Fuente, ${ }^{1}$ \\ Hennessey M. Sabanal, ${ }^{1}$ Alison Paolo N. Bareng, ${ }^{1}$ Inez Andrea P. Medado, ${ }^{3}$ Edelwisa S. Mercado, ${ }^{3}$ Mario S. Baquilod, ${ }^{4}$ \\ Jennifer S. Luchavez, ${ }^{1}$ Julius Clemence R. Hafalla, ${ }^{2}$ Chris J. Drakeley, ${ }^{2}$ and Fe Esperanza J. Espino ${ }^{1}$ \\ ${ }^{1}$ Department of Parasitology, Research Institute for Tropical Medicine, Muntinlupa, Philippines; ${ }^{2}$ Faculty of Infectious and Tropical Diseases, \\ London School of Hygiene and Tropical Medicine, London, United Kingdom; ${ }^{3}$ Molecular Biology Laboratory, Research Institute for Tropical \\ Medicine, Muntinlupa, Philippines; ${ }^{4}$ Department of Health, MIMAROPA Center for Health Development, Quirino Memorial Medical Center
} Compound, Quezon, Philippines

\begin{abstract}
Following substantial progress in malaria control in the Philippines, new surveillance approaches are needed to identify and target residual malaria transmission. This study evaluated an enhanced surveillance approach using rolling cross-sectional surveys of all health facility attendees augmented with molecular diagnostics and geolocation. Facility surveys were carried out in three sites representing different transmission intensities: Morong, Bataan (pre-elimination), Abra de llog, Occidental Mindoro (stable medium risk), and Rizal, Palawan (high risk, control). Only one rapid diagnostic test (RDT)-positive infection and no PCR confirmed infections were found in Bataan and Occidental Mindoro, suggesting the absence of transmission. In Palawan, the inclusion of all health facility attendees, regardless of symptoms, and use of molecular diagnostics identified 313 infected individuals in addition to 300 cases identified by routine screening of febrile patients with the RDT or microscopy. Of these, the majority (313/613) were subpatent infections and only detected using molecular methods. Simultaneous collection of GPS coordinates on tablet-based applications allowed real-time mapping of malaria infections. Risk factor analysis showed higher risks in children and indigenous groups, with bed net use having a protective effect. Subpatent infections were more common in men and older age-groups. Overall, malaria risks were not associated with participants' classification, and some of the non-patient clinic attendees reported febrile illnesses $(1.9 \%, 26 / 1,369)$, despite not seeking treatment, highlighting the widespread distribution of infection in communities. Together, these data illustrate the utility of health facility-based surveys to augment surveillance data to increase the probability of detecting infections in the wider community.
\end{abstract}

\section{INTRODUCTION}

The Philippines declared its vision of eliminating malaria by 2030 with a goal of reducing malaria incidence in the country by $90 \%$ relative to a 2016 baseline of 6,604 reported cases. Through its strategy of subnational elimination, enhanced case detection and treatment, and vector control, the country aims to increase the number of malaria-free provinces from 32 to 74 by 2022 out of the 81 provinces. ${ }^{1,2}$ However, malaria continues to be a public health burden with highly variable transmission across the country. In 2018, 4,902 indigenous cases and 1 death were reported with approximately $95 \%$ of these on Palawan island (Annual Parasite Index $\geq 1$ per 1,000 at-risk population). Within Palawan, transmission is geographically heterogeneous, with malaria-free municipalities in the north, and southern municipalities endemic for all five human Plasmodium species.

Recent WHO guidelines on malaria surveillance define surveillance as a core intervention required in settings of any level of transmission to meet elimination goals. The guidelines also highlighted the need for increasingly spatial and temporal resolution data on malaria infection as transmission declines. ${ }^{3}$ Although population-based community surveys remain the gold standard for measuring prevalence and assessing spatial patterns of infection, these sampling approaches are highly resource-intensive and may require prohibitively large sample sizes in low-transmission settings. Alternatively, surveys of

\footnotetext{
*Address correspondence to Ralph A. Reyes, Department of Parasitology, Research Institute for Tropical Medicine, 9002 Research Dr., Filinvest Corporate City, Muntinlupa 1781, Philippines. E-mail: rreyes.rmt@gmail.com
}

easy access groups, such as health facility attendees or school children, can be used to provide rapid estimates of malaria prevalence within the community (e.g., refs. 4-8). These surveys may not fully capture the distribution of infection in the entire population but are operationally feasible and cost-effective to implement. As malaria transmission decreases, spatial heterogeneity becomes more pronounced, with substantial variations observed in the geographic distribution of infections. ${ }^{9}$ However, by incorporating methods of geolocating participant households using tablet-based applications, fine-scale maps of malaria infection can be created in near real time, allowing identification of foci of transmission ${ }^{10}$ which are relevant for areas like Palawan.

In addition, conventional diagnostic methods recommended by the WHO have limitations for surveillance because low parasite density resulting in submicroscopic and asymptomatic infections is missed. ${ }^{11,12}$ With only symptomatic infections being tested, individuals who are not seeking treatment are overlooked, and malaria transmission estimates based on clinical cases reporting to health facilities are biased. ${ }^{13}$ Asymptomatic and subpatent infections comprise most of the malaria infections in low endemic areas, despite adequate malaria control measures, and contribute to maintaining transmission, undermining elimination efforts. ${ }^{14}$ Most of these infections are not detectable by conventional microscopy or rapid diagnostic tests (RDTs), necessitating the use of molecular techniques. ${ }^{15,16}$ Detecting these infections can be challenging because of the infrequent reports of clinical cases and the low probability of identifying infections. For example, in the neighboring country Malaysia, in the state of Sabah, 54 Plasmodium infections were identified from a community-based survey, including 876 individuals, despite 
no microscopically positive malaria cases or clinical reports during this time period. ${ }^{17}$ Also, in Asia-Pacific countries, reliance on passive health facility surveillance does not detect asymptomatic infections. ${ }^{18}$

To assess how health facility-based surveys with molecular diagnostics could be used to support malaria elimination efforts, we conducted rolling cross-sectional surveys in the provinces of Palawan, Occidental Mindoro, and Bataan, three areas of the Philippines with different levels of reported transmission. The overall aims were to 1) develop methods for health facility-based surveys applying improved diagnostics and geolocation technologies, 2) assess the utility of enhanced surveillance approaches to improve detection of malaria infections, and 3) identify characteristics of individuals with subpatent infections.

\section{METHODS}

Study areas. The areas were selected based on the 2014 Philippines' National Malaria Program operational definition of malaria-endemic provinces (Figure 1). In that year, Palawan, Occidental Mindoro, and Bataan were categorized as stable high risk or control phase, stable medium risk of transmission, and malaria pre-elimination provinces, respectively, and in the same order, the annual parasitological indices per 1,000 population were $0,0.35$, and 5.7, respectively, in $2018 .{ }^{19}$ Demographic information and land areas of the selected areas are shown in Table 1. According to the Philippine Statistics Authority Census of 2000 , the population in all study sites comprised various ethnicities and indigenous groups. In Palawan, almost half of the population belongs to different indigenous groups. The Palaw'an indigenous group comprises

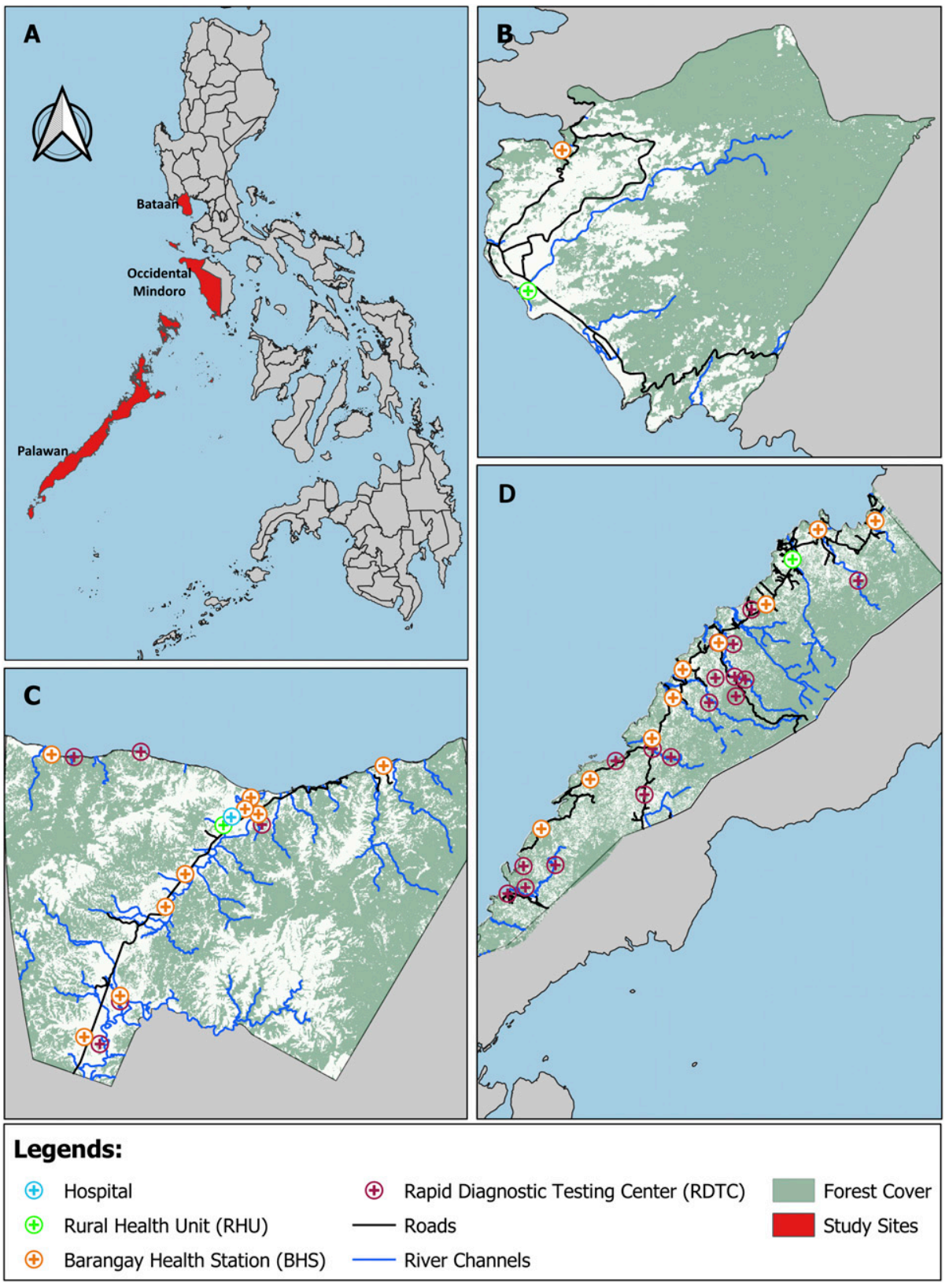

FIGURE 1. Study sites and surveyed health facilities. This figure appears in color at www.ajtmh.org. 
TABLE 1

Description of study sites

\begin{tabular}{|c|c|c|c|c|}
\hline & Bataan, Bataan (A) & Occidental Mindoro, Occidental Mindoro (B) & \multicolumn{2}{|c|}{ Palawan, Palawan (C) } \\
\hline Land area & $219.20 \mathrm{~km}^{2}$ & $533.70 \mathrm{~km}^{2}$ & \multicolumn{2}{|c|}{$1,256.47 \mathrm{~km}^{2}$} \\
\hline Population density & $135.40 / \mathrm{km}^{2}$ & $58.67 / \mathrm{km}^{2}$ & \multicolumn{2}{|c|}{$39.87 / \mathrm{km}^{2}$} \\
\hline $\begin{array}{l}\text { Transmission setting } \\
\text { category (DoH, 2014) }\end{array}$ & Pre-elimination & Stable medium risk & \multicolumn{2}{|c|}{ Stable high/control } \\
\hline $\begin{array}{l}\text { Annual parasite incidence } \\
\text { in } 2013(\mathrm{DoH} 2018)\end{array}$ & $\begin{array}{l}\text { No indigenous malaria reported } \\
\text { since } 2011\end{array}$ & 0.35 & \multicolumn{2}{|c|}{5.7} \\
\hline Sampling dates & May 2017-March 2018 & July 2017-June 2018 & $\begin{array}{c}\text { (Year 1) } \\
\text { Jun 2016-June } 2017\end{array}$ & $\begin{array}{c}\text { (Year 2) } \\
\text { Jul 2017-June } 2018\end{array}$ \\
\hline Sampling frequency & 1 Week bimonthly & 1 Week bimonthly & 1 Week monthly & 1 Week monthly \\
\hline No of barangays covered & $2 / 5$ & All 10 & All 11 & $5 / 11$ \\
\hline Number of health facilities & $n=2$ & $n=17$ & $n=27$ & $n=4$ \\
\hline Rural health unit & 1 & 1 & 1 & 1 \\
\hline Barangay health station & 1 & 9 & 10 & - \\
\hline RDT center & - & 6 & 16 & 3 \\
\hline Hospital & - & 1 & - & - \\
\hline
\end{tabular}

$38.7 \%$ of the total population in Palawan; other ethnic groups include Kagayanen (1.9\%), Tausug (1.8\%), Cuyunon (1.2\%), Maranao (1.1\%), Jama Mapun (0.9\%), and Agutaynen $(0.1 \%){ }^{20}$ The main occupations include subsistence agriculture, swidden agriculture (slash-and-burn), and fishing. In Occidental Mindoro, $64.2 \%$ of the population comprises Tagalog and $30.6 \%$ of indigenous groups, and the majority of the population in Bataan classified themselves as Tagalog (91.0\%), with $0.8 \%$ of indigenous population. ${ }^{21,22}$ Residents in Occidental Mindoro and Bataan are primarily long-time settlers with small businesses. All provinces are predominantly rural, partly forested with seasonal rainfall generally from May to October. Primary healthcare services are provided by the rural health unit (RHU) and barangay health stations. In addition to these facilities and service remote communities, Occidental Mindoro and Palawan have malaria testing (using RDTs) and treatment centers based at households of community health workers. With supervision from $\mathrm{RHU}$ staff, community volunteers operate the barangay health stations and remote malaria testing and treatment centers.

Study design and sampling. Rolling cross-sectional surveys in the health facilities were carried out every first week of the month for 2 years in Palawan. During the first year of the project (June 2016-June 2017), surveys were conducted in the 27 health facilities in the municipality (Table 1). Data collection was extended to a second year (July 2017-June 2018), with surveys limited to the rural health center and the three malaria RDT centers that reported the highest numbers of cases the previous year. In Occidental Mindoro and Bataan, these surveys were conducted the first week every 2 months over a 12-month period. Seventeen health facilities were surveyed in Occidental Mindoro. These were the RHUs, one district hospital, nine barangay health stations, and six RDT centers. In Bataan, information was collected from the RHUs and one barangay health station. Nearby hospitals are accessible to the residents of Occidental Mindoro and Bataan, unlike in Palawan. Hence, residents typically opt to send their patients to these hospitals. The distance from Dr. Jose Rizal District Hospital from nearest to farthest barangay ranges from $13.7 \mathrm{~km}$ to $64.9 \mathrm{~km}$ by road. The southernmost barangays, Latud and Canipaan, were excluded because they are not accessible by road. Moreover, the Rio Tuba Nickel Mining Corporation Hospital in Bataraza, Palawan, is $72.1 \mathrm{~km}$ away from Palawan.
Health facility staff underwent training on study procedures including obtaining written informed consent, malaria blood film and blood spot preparation, collection of geolocation information of participant's residence, and history of illness and travel. Questionnaire data and GPS coordinates of participant households were collected using GeoODK (GeoMarvel, Alexandria, VA) on Android tablets using satellite imagery and known landmarks to geolocate households; this methodology is described in detail by Fornace et al. ${ }^{10}$ This included basic demographic information, symptoms, axillary temperature, movement history, malaria prevention practices, and initial RDT results. Activities and travel history within and beyond the study sites in the past 7 days were recorded. Participants, without age restrictions, were classified as either patients, that is, individuals seeking health consultations were referred, or companions, that is, those who accompany the patients. Women in the maternal clinic and individuals with serious illnesses who required urgent care or transport to higher level health facilities and individuals who have resided in the study sites less than 7 days were excluded.

Research ethics. The Research Institute for Tropical Medicine-Institutional Review Board (IRB no.: 2016-04), and London School of Hygiene and Tropical Medicine (11597) approved this study.

Assessment of malaria infection. Health facility workers collected finger-prick blood samples for malaria blood film microscopy and three $20-\mu \mathrm{L}$ spots on filter papers (3MM, Whatman, Maidstone, United Kingdom). Filter papers were dried and stored with desiccants at $-20^{\circ} \mathrm{C}$. Thick and thin blood films were examined by trained malaria microscopists from the Research Institute for Tropical Medicine, supervising the health facility workers with all positive slides and $10 \%$ of the negative slides validated by a WHO-certified level one malaria microscopist. All participants from Palawan and Occidental Mindoro were also tested for malaria using the SD Bioline Malaria RDT, with either Ag Pf/Pan ${ }^{\mathrm{TM}}$ or $\mathrm{Ag} \mathrm{Pf} / \mathrm{Pv}^{\mathrm{TM}}$ (Abbott Rapid Diagnostics, Santa Clara, CA) depending on its availability in the market. Individuals tested RDT- and/or microscopy-positive were treated on-site by the health facility personnel following the Philippines' national treatment guidelines for malaria.

DNA was extracted from approximately $10 \mu \mathrm{L}$ of dried blood spots (DBS) on filter papers using the Chelex 100 (Bio-Rad Laboratories, Hercules, CA) method $^{23}$ modified to $6 \%$. A 
nested PCR assay targeting Plasmodium sp. small subunit ribosomal RNA genes was used to identify positive samples, and species-specific primers were used on genus-positive samples. ${ }^{24-27}$ Results were visualized on $2 \%$ agarose gel. This malaria diagnosis by PCR has a limit of detection of 0.2 parasites/ $\mu \mathrm{L}$. A subset of samples was extracted using a Qiagen DNA Mini Kit (Qiagen, Hilden, Germany) to validate results. All samples were tested with PCR, regardless of RDT and microscopy results; positive results were referred to as malaria infections. Within this study, we defined patent infections as individuals positive with both PCR and microscopy and/or RDT, and infections were classified as subpatent if they could only be identified by PCR and were negative for microscopy and/or RDT.

Data management and analysis. Each participant was assigned a unique ID to enable linkage to samples. Data for geolocation of residence were collected during the interview using a designed electronic questionnaire run on GeoODK application. Participants were asked to locate their homes by pointing to its location on Android tablets. All information was later sent to the project's secure cloud server. Households with missing GPS coordinates due to limited tablet availability during the first quarter of the study were visited and located using a handheld GPS (Garmin Ltd., Olathe, KS). ${ }^{10}$ Microscopy, RDT, and PCR results were recorded in the laboratory worksheets and were double encoded using Microsoft ${ }^{\circledR}$ Excel $^{\circledR} 2016$ (Microsoft Corporation, Redmond, WA) and were merged with questionnaire results. Results of malaria blood film microscopy/RDT and malaria PCR were plotted on QGIS ${ }^{\mathrm{TM}}$ Desktop software version 3.8.2 (QGIS.org, Open Source Geospatial Foundation Project). ${ }^{28}$

All data sets were analyzed using $R$ statistical programming language version 3.6.3 (R Foundation for Statistical Computing, Vienna, Austria). ${ }^{29}$ Individuals with insufficient samples and incomplete questionnaire data needed for the analysis of this study were excluded. To compare the sociodemographic characteristics of patients with other individuals screened by health facility surveys, we fit a binomial generalized linear mixed model with participant status as the outcome. Data were included from all sites with health facility included as a random effect. For Palawan only, binomial generalized mixed models were used to identify risk factors for malaria infection including a random effect for the health facility of the survey. An additional model was developed to determine the probability of patent infection (defined as infections detected by microscopy or RDTs) from all infected individuals. To select variables for inclusion, univariate analyses were conducted, with all variables with $P<0.2$ screened for inclusion in multivariate analyses. The final multivariate analyses were fit in a forward-stepwise manner, with variables included in the final model with $P<0.05$.

\section{RESULTS}

Characteristics of study sites and population demographics. The distribution of participants by study site, nature of the visit to health facility (i.e., patient or a patient's companion), gender, median age, and presence of fever is summarized in Supplemental Table S1. Out of 5,746 participants in Palawan, only 5,620 were included in the analysis due to sample insufficiency $(n=24)$ and incomplete questionnaire data $(n=$ 102). Most of the participants in all sites were patients, rather than companions. There were higher proportions of female participants in all sites, with the most notable difference observed in Bataan and Occidental Mindoro. These two sites also had much older age-groups and lower proportions of febrile individuals than Palawan. A review of records disclosed that in 2018, $70.8 \%$ and $61.6 \%$ of the consultations in Bataan and Occidental Mindoro, respectively, were for acute respiratory infections and could reflect mothers accompanying their children.

High proportions of health facility attendees were the Palaw'an indigenous people in both the first $(63.4 \%, n=3,659)$ and second $(46.1 \%, n=523)$ years of surveillance in Palawan. By contrast, clinic attendees were primarily Tagalog, the nonindigenous group, at health facilities surveyed in Occidental Mindoro (56.4\%, $n=999)$ and Bataan $(97.3 \%, n=872)$, whereas the Tagalog attendees in Palawan were 9.1\% (524) in the first year and $20.2 \%$ (229) in the second year. On the other hand, only $0.4 \%$ (4) from the aboriginal group in Bataan (Aetas) and $41.6 \%$ (738) in Occidental Mindoro (Mangyans) attended the health facilities. Remaining attendees identified themselves as migrants or not originally from the province, with $27.5 \%(1,584)$ in the first year and $33.7 \%$ (383) in Palawan, $2.0 \%$ (35) in Occidental Mindoro, and 2.2\% (20) in Bataan; these individuals had been residing in the area for more than 7 days before the survey.

For all study participants, we compared the association of sociodemographic characteristics of individuals classified as patients $(6,458 / 8,288)$ and companions $(1,830)$. Companions were more likely to be adults, females, indigenous groups, and forest workers (Table 2).

Malaria infection in patients and companions. Malaria infections were detected only in Palawan either by RDTs/ microscopy or PCR. All samples from Occidental Mindoro and Bataan tested PCR-negative (Supplemental Table S2). Although one RDT-positive individual was detected in Occidental Mindoro, this was confirmed to be PCR-negative, suggesting a false-positive RDT result or historical exposure. In the first year of collection in Palawan, there was twice the number of individuals whose PCR results were positive for malaria compared with microscopy and RDTs. It was noteworthy that $12.9 \%(n=1,354)$ of companions were positive for malaria infections by PCR, contributing $28.5 \%$ (175/613) of all positive cases. PCR increased the detected malaria infections in patients by $36.7 \%$ (254/693) compared with microscopy and $38 \%$ (268/706) compared with RDT. Enhancing the surveillance through PCR testing and adding companions increased the total infections from $6.2 \%(255 / 4,095)$ by microscopy and $6.1 \%(268 / 4,391)$ by RDT to $10.7 \%(613 /$ $5,722)$.

In the second year of collection, 20.1\% $(n=228)$ of individuals were malaria positive by PCR as compared with $8.2 \%$ and $8.7 \%$ of microscopy and RDTs, respectively (Supplemental Table S2). Comparing the two phases of surveillance, the second year of collection from the four health facilities that reported the highest malaria cases confirms that the proportion of PCR positives among companions (23.9\%, 38/159) is high like year $1(17.8 \%, 52 / 292)$ but higher than other facilities $(11.6 \%, 123 / 1,062)$.

Plasmodium species identified. Within Palawan, Plasmodium falciparum was the most common species detected using blood film microscopy $(74.3 \%, 223 / 300)$ followed by Plasmodium vivax $(18.0 \%, n=54)$, Plasmodium malariae (1.3\%, $n=4)$, and mixed infections $(6.0 \%, n=18)$; this was similar in year $2(76.9 \%, 70 / 91 ; 11.0 \% . n=103.3 \%, n=3$; 
TABLE 2

Sociodemographic factors associated with participant classification (patients vs. companions)

\begin{tabular}{|c|c|c|c|c|c|c|c|}
\hline \multirow[b]{2}{*}{ Variable $(n=8,288)$} & \multirow[b]{2}{*}{ N } & \multicolumn{3}{|c|}{ Unadjusted } & \multicolumn{3}{|c|}{ Adjusted } \\
\hline & & OR & $95 \% \mathrm{Cl}$ & $P$-value & OR & $95 \% \mathrm{Cl}$ & $P$-value \\
\hline Age (years) & & & & $<0.001$ & & & $<0.001$ \\
\hline Under 5 & 1,574 & - & - & & - & - & \\
\hline $5-10$ & 1,182 & 0.95 & $(0.77-1.17)$ & & 0.80 & $(0.64-1.01)$ & \\
\hline $11-20$ & 1,373 & 0.71 & $(0.58-0.86)$ & & 0.59 & $(0.46-0.75)$ & \\
\hline $21-30$ & 1,284 & 0.39 & $(0.32-0.48)$ & & 0.39 & $(0.30-0.49)$ & \\
\hline Over 30 & 2,875 & 0.46 & $(0.39-0.55)$ & & 0.45 & $(0.36-0.55)$ & \\
\hline Gender & & & & $<0.001$ & & & $<0.001$ \\
\hline Female & 5,026 & - & - & & - & - & \\
\hline Male & 3,262 & 1.64 & $(1.45-1.84)$ & & 1.34 & $(1.18-1.53)$ & \\
\hline Ethnicity & & & & $<0.001$ & & & $<0.001$ \\
\hline Other ethnicity & 1,623 & - & - & & - & - & \\
\hline Indigenous groups & 4,271 & 0.48 & $(0.41-0.57)$ & & 0.53 & $(0.44-0.64)$ & \\
\hline Tagalog & 2,394 & 1.07 & $(0.82-1.39)$ & & 1.05 & $(0.80-1.38)$ & \\
\hline Occupation & & & & & & & \\
\hline 1. Agriculture & & & & 0.408 & & & - \\
\hline No & 7,036 & _- & _ & & - & - & \\
\hline Yes & 1,252 & 0.70 & $(0.60-0.82)$ & & - & - & \\
\hline 2. Forestry & & & & 0.016 & & & 0.022 \\
\hline No & 7,572 & - & - & & - & - & \\
\hline Yes & 716 & 0.50 & $(0.41-0.61)$ & & 0.77 & $(0.61-0.96)$ & \\
\hline 3. Business owner & & & & $<0.001$ & & & 0.003 \\
\hline No & 7,803 & - & - & & - & - & \\
\hline Yes & 485 & 1.90 & $(1.41-2.56)$ & & 1.61 & $(1.16-2.24)$ & \\
\hline 4. Unemployed & & & & $<0.001$ & & & $<0.001$ \\
\hline No & 6,027 & _- & _ & & - & - & \\
\hline Yes & 2,261 & 0.49 & $(0.43-0.56)$ & & 0.71 & $(0.60-0.85)$ & \\
\hline Education & & & & 0.004 & & & 0.004 \\
\hline None & 3,236 & - & - & & - & - & \\
\hline Primary & 1851 & 1.13 & $(1.00-1.29)$ & & 1.31 & $(1.12-1.54)$ & \\
\hline Secondary & 3,201 & 1.07 & $(0.90-1.26)$ & & 1.24 & $(1.00-1.55)$ & \\
\hline
\end{tabular}

$4.4 \%, n=4$, respectively). Remaining blood films were positive for malaria but, because of poor thin smears, not speciated (year 1, $n=1$; year 2, $n=4$ ). By PCR, all five species of malaria were detected. The observations were similar, with $P$. falciparum being the most prevalent species $(49.9 \%, n=$ $306 / 613)$, followed by $P$. vivax $(12.2 \%, n=75), P$. malariae $(4.7 \%, n=29)$, Plasmodium ovale $(0.3 \%, n=2)$, Plasmodium knowlesi $(0.2 \%, n=1)$, and mixed infections $(8.0 \%, n=49)$. However, 153 samples positive for Plasmodium were not speciated because of sample insufficiency. Likewise, PCR results in year 2 showed $P$. falciparum $(55.3 \%, n=126 / 228)$ as the most dominant species, followed by $P$. vivax $(11.8 \%, n=$ 27), P. malariae (1.3\%, $n=3)$, and mixed (9.7\%, $n=22)$. Similar to year 1 , species identification of 50 positives for Plasmodium were not performed (Supplemental Table S3).

In the first year of surveillance, there was $82.9 \%$ (179/216) agreement on the species of the parasite present in samples that were positive by both PCR and microscopy.
Consequently, a low proportion of all positive samples by microscopy $(15.8 \%, 42 / 265)$ were negative by PCR and a high proportion of positive samples by PCR $(58.5 \%, 314 / 537)$ were negative by microscopy (Table 3 ). With microscopy, $74.3 \%$ (142/191) were correctly identified as $P$. falciparum and $62.2 \%$ $(28 / 45)$ for $P$. vivax. All $P$. malariae infections $(100.0 \%, 4 / 4)$ were accurately diagnosed. Plasmodium ovale infections were misidentified as $P$. falciparum (1) and $P$. vivax (1).

Seasonal and spatial distribution of malaria infections. For the first year of surveillance in Palawan, the number of malaria infection and individuals attending health facilities varied monthly (Figure 2). Although there was seasonality in the number of patients attending health facilities and the total number of infections, there were some temporal trends in the proportions of individuals detected as positive by either routine or enhanced surveillance. Although the rainfall season is from May to October, increased malaria infections were only noted in July and August.

TABLE 3

Comparison of PCR and malaria microscopy for active health facility survey

\begin{tabular}{|c|c|c|c|c|c|c|c|c|}
\hline \multirow[b]{2}{*}{ Microscopy result } & \multicolumn{8}{|c|}{ No. of samples with the following result by PCR (\%) } \\
\hline & P. falciparum & P. vivax & P. malariae & P. ovale & Plamodium knowlesi & Mixed $^{*}$ & Negative & Total \\
\hline P. falciparum & $142(74.3)$ & $2(1.0)$ & $1(0.5)$ & $1(0.5)$ & $0(0.0)$ & $11(5.8)$ & $34(17.8)$ & 191 \\
\hline P. vivax & $5(11.1)$ & $28(62.2)$ & $0(0.0)$ & $1(2.2)$ & $0(0.0)$ & $5(11.1)$ & $6(13.3)$ & 45 \\
\hline P. malariae & $0(0.0)$ & $0(0.0)$ & $4(100.0)$ & $0(0.0)$ & $0(0.0)$ & $0(0.0)$ & $0(0.0)$ & 4 \\
\hline Mixed $\dagger$ & $6(35.3)$ & $4(23.5)$ & $1(5.9)$ & $0(0.0)$ & $0(0.0)$ & $5(29.4)$ & $1(5.9)$ & 17 \\
\hline Negative & $130(2.78)$ & $37(0.8)$ & $17(0.4)$ & $0(0.0)$ & $1(0.0)$ & $22(0.5)$ & $4,476(95.6)$ & 4,683 \\
\hline Total & $283(5.7)$ & $71(1.4)$ & $23(0.5)$ & $2(0.0)$ & $1(0.0)$ & $43(0.9)$ & $4,518(91.4)$ & 4,941 \\
\hline
\end{tabular}

$P$. falciparum $=$ Plasmodium falciparum $P$. malariae $=$ Plasmodium malariae $; P$. ovale $=$ Plasmodium ovale $; P$. vivax = Plasmodium vivax

${ }^{*}$ Mixed infections by PCR: P. falciparum/P. vivax, P. falciparum/P. ovale, P. falciparum/P. malariae, P. vivax/P. malariae, P. falciparum/P. vivax/P. malariae.

$\dagger$ Mixed infections by microscopy: $P$. falciparum/P. vivax and $P$. falciparum/P. malariae. 


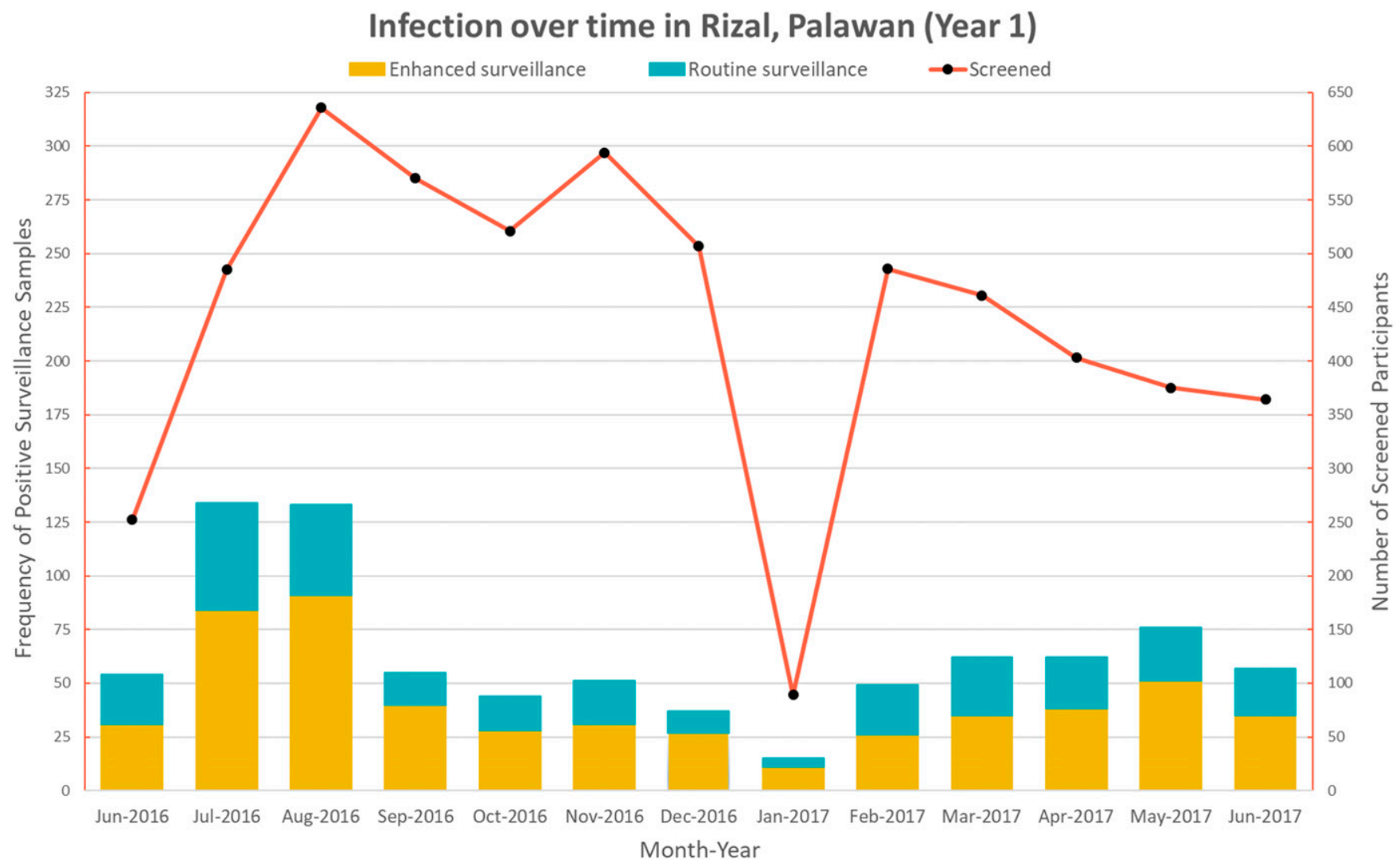

FIgURE 2. Temporal trend in Rizal, Palawan. This figure appears in color at www.ajtmh.org.

Figure 3 shows the difference in spatial distributions of infections detected by both surveillance approaches. Although this analysis shows the utility of health facility surveys using this platform to capture real-time spatial data on the locations of participants' residences, further analysis of spatial patterns of health facility attendance and infections was explored by Fornace et al. ${ }^{30}$

Factors associated with malaria infections. As malaria infections were only identified within Palawan and the first year of surveillance represented the most comprehensive data set, we chose to focus on risk factor analysis on these data. Within this year, the inclusion of malaria screening of all companions increased the identification of patent infections by $16.6 \%$ ( $n=$ $60 / 361)$. This further improved to $18.5 \%(n=125 / 676)$ when PCR was used to assess infection. Subsequent risk factor analysis showed that the odds of malaria infection (as detected by any diagnostic methods, $n=5,620$ ) were almost three times higher in 11- to 20-year age-group than older than 30-year age-group (Table 4). In addition, male individuals, Palaw'an indigenous group, and individuals sleeping without
A

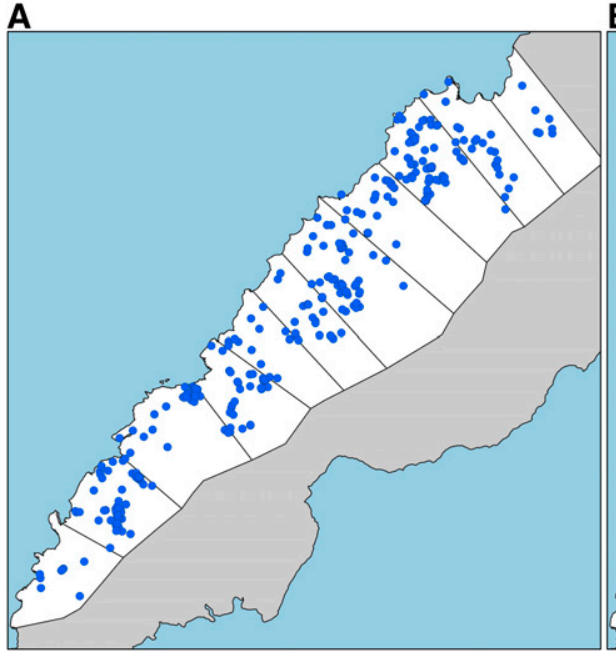

B

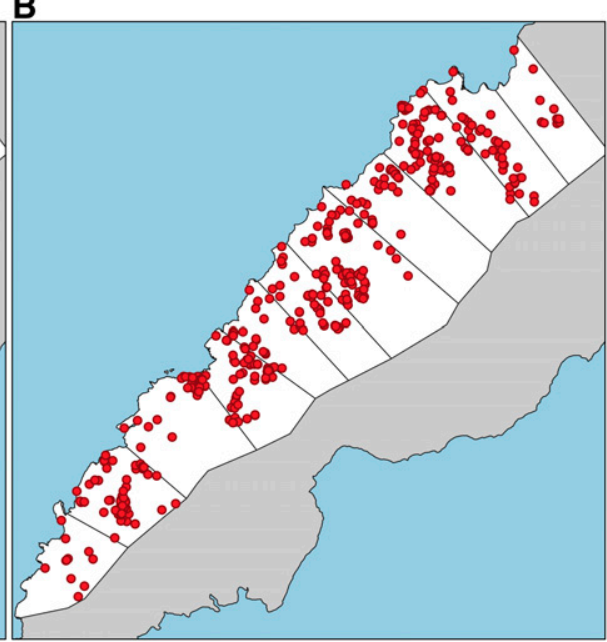

FIGURE 3. Malaria surveillance approaches; (A) routine surveillance, (B) enhanced surveillance. This figure appears in color at www.ajtmh.org. 
bed nets had higher risks of infection. A significantly higher infection risk was observed in individuals with lower education levels; however, there was no clear association with specific occupational activities. There was no significant difference in infection risks detected between patients or companions screened.

For all malaria cases, we compared the risks of the patent (356/669) and subpatent malaria (313). Patent malaria infections were more common in younger age-groups, with risks of patent infections decreasing with age (Table 5). Male gender had almost twice the odds of patent infections compared with female gender. Companions were more likely to have subpatent infections, as would be expected considering they were not seeking treatment. No associations between bed net use or history of travel and patent infections were identified.

\section{DISCUSSION}

We developed an enhanced surveillance approach to demonstrate the utility of health facility surveys in low- and high-transmission settings incorporated with both molecular diagnostics and geolocation. The inclusion of companions and PCR testing provided additional information to assess transmission levels in the catchment populations that would not have been possible with the standard malaria surveillance system and captured wider demographic groups. The use of PCR led to about $58 \%$ increase in the total number of infections detected from 255 samples by microscopy and 268 by RDTs to 438 . The simultaneous collection of spatial data and the use of geographic information systems further increase the resolution of the spatial distribution of malaria infection. This approach can provide an operationally feasible method to supplement existing health facility data to improve

TABLE 4

Factors associated with Plasmodium infection detectable by PCR

\begin{tabular}{|c|c|c|c|c|c|c|c|}
\hline \multirow[b]{2}{*}{ Variable $(n=5,620)$} & \multirow[b]{2}{*}{ N } & \multicolumn{3}{|c|}{ Unadjusted } & \multicolumn{3}{|c|}{ Adjusted } \\
\hline & & OR & $95 \% \mathrm{Cl}$ & $P$-value & $\mathrm{OR}$ & $95 \% \mathrm{Cl}$ & $P$-value \\
\hline Age (years) & & & & $<0.001$ & & & $<0.001$ \\
\hline Under 5 & 1,323 & - & - & & - & - & \\
\hline $5-10$ & 935 & 1.55 & $(1.19-2.03)$ & & 2.10 & $(1.56-2.83)$ & \\
\hline $11-20$ & 977 & 1.59 & (1.22-2.07) & & 2.64 & (1.92-3.64) & \\
\hline $21-30$ & 737 & 1.18 & $(0.87-1.60)$ & & 1.61 & (1.15-2.24) & \\
\hline Over 30 & 1,648 & 0.72 & $(0.54-0.93)$ & & 0.96 & $(0.72-1.28)$ & \\
\hline Gender & & & & $<0.001$ & & & $<0.001$ \\
\hline Female & 3,230 & - & - & & - & - & \\
\hline Male & 2,390 & 1.41 & $(1.19-1.68)$ & & 1.49 & $(1.24-1.79)$ & \\
\hline Ethnicity & & & & $<0.001$ & & & $<0.001$ \\
\hline Other ethnicity & 1,581 & - & - & & - & - & \\
\hline Palaw'an & 3,516 & 4.20 & $(3.16-5.58)$ & & 3.87 & $(2.86-5.23)$ & \\
\hline Tagalog & 523 & 1.13 & $(0.67-1.93)$ & & 1.13 & $(0.66-1.94)$ & \\
\hline Occupation & & & & & & & \\
\hline 1. Agriculture & & & & 0.079 & & & - \\
\hline No & 4,633 & - & - & & & - & \\
\hline Yes & 987 & 0.80 & $(0.63-1.03)$ & & & - & \\
\hline 2. Forestry & & & & 0.680 & & & - \\
\hline No & 5,094 & - & - & & - & - & \\
\hline Yes & 526 & 0.94 & $(0.70-1.26)$ & & - & - & \\
\hline 3. Business owner & & & & 0.815 & & & - \\
\hline No & 5,422 & - & - & & - & - & \\
\hline Yes & 198 & 0.94 & $(0.56-1.58)$ & & - & - & \\
\hline 4. Unemployed & & & & $<0.001$ & & & - \\
\hline No & 4,740 & - & - & & - & - & \\
\hline Yes & 880 & 0.61 & $(0.46-0.82)$ & & - & - & \\
\hline Activities outside house* & & & & 0.328 & & & - \\
\hline No & 2,724 & - & - & & - & - & \\
\hline Yes & 2,896 & 1.09 & $(0.91-1.31)$ & & - & - & \\
\hline History of travel & & & & 0.267 & & & - \\
\hline No & 4,303 & - & - & & - & - & \\
\hline Yes & 1,317 & 0.87 & $(0.68-1.11)$ & & - & - & \\
\hline Type of participant & & & & 0.201 & & & - \\
\hline Patient & 4,286 & - & - & & - & - & \\
\hline Companion & 1,334 & 1.14 & $(0.93-1.39)$ & & - & - & \\
\hline Education & & & & $<0.001$ & & & $<0.001$ \\
\hline None & 2,738 & - & - & & - & - & \\
\hline Primary & 2,151 & 0.78 & $(0.65-0.94)$ & & 0.66 & $(0.53-0.83)$ & \\
\hline Secondary & 731 & 0.42 & $(0.29-0.60)$ & & 0.59 & (0.39-0.89) & \\
\hline Bed net use & & & & $<0.001$ & & & $<0.001$ \\
\hline Yes & 5,485 & - & - & & - & - & \\
\hline No & 135 & 3.89 & $(2.58-5.89)$ & & 3.50 & $(2.28-5.38)$ & \\
\hline Health facility type & & & & 0.048 & & & - \\
\hline Barangay health station & 2,197 & - & - & & - & - & \\
\hline Rural health unit & 1,091 & 0.95 & $(0.26-3.45)$ & & - & - & \\
\hline Rapid diagnostic testing center & 2,332 & 1.99 & $(1.17-3.41)$ & & - & - & \\
\hline
\end{tabular}

*Activities include child play, school, and work-related activities. 
TABLE 5

Factors associated with Plasmodium infection detectable by RDTs/microscopy, among all infections identified by PCR

\begin{tabular}{|c|c|c|c|c|c|c|c|}
\hline \multirow[b]{2}{*}{ Variable $(n=669)$} & \multirow[b]{2}{*}{ N } & \multicolumn{3}{|c|}{ Unadjusted } & \multicolumn{3}{|c|}{ Adjusted } \\
\hline & & OR & $95 \% \mathrm{Cl}$ & $P$-value & OR & $95 \% \mathrm{Cl}$ & $P$-value \\
\hline Age (years) & & & & $<0.001$ & & & $<0.001$ \\
\hline Under 5 & 140 & - & - & & - & - & \\
\hline $5-10$ & 142 & 0.81 & $(0.49-1.18)$ & & 0.86 & $(0.51-1.46)$ & \\
\hline $11-20$ & 168 & 0.54 & $(0.19-0.52)$ & & 0.66 & $(0.40-1.08)$ & \\
\hline $21-30$ & 88 & 0.28 & $(0.12-0.39)$ & & 0.43 & $(0.23-0.80)$ & \\
\hline Over 30 & 131 & 0.24 & $(0.13-0.61)$ & & 0.29 & $(0.17-0.50)$ & \\
\hline Gender & & & & $<0.001$ & & & $<0.001$ \\
\hline Female & 327 & - & - & & - & - & \\
\hline Male & 342 & 2.24 & $(1.63-3.09)$ & & 1.99 & $(1.42-2.79)$ & \\
\hline Ethnicity & & & & 0.151 & & & - \\
\hline Other ethnicity & 72 & - & - & & - & - & \\
\hline Palaw'an & 575 & 1.50 & $(0.89-2.55)$ & & - & - & \\
\hline Tagalog & 22 & 0.78 & $(0.28-2.17)$ & & - & - & \\
\hline Occupation & & & & & & & \\
\hline 1. Agriculture & & & & 0.015 & & & - \\
\hline No & 566 & - & - & & - & - & \\
\hline Yes & 103 & 0.58 & $(0.37-0.90)$ & & - & - & \\
\hline 2. Forestry & & & & 0.006 & & & - \\
\hline No & 597 & - & - & & - & - & \\
\hline Yes & 72 & 0.48 & $(0.28-0.81)$ & & - & - & \\
\hline 3. Business owner & & & & 0.754 & & & - \\
\hline No & 650 & - & - & & - & - & \\
\hline Yes & 19 & 1.16 & $(0.45-3.02)$ & & - & - & \\
\hline 4. Unemployed & & & & $<0.001$ & & & - \\
\hline No & 606 & - & - & & - & - & \\
\hline Yes & 63 & 0.27 & $(0.15-0.49)$ & & - & - & \\
\hline Activities outside house* & & & & 0.015 & & & - \\
\hline No & 305 & - & - & & - & - & \\
\hline Yes & 364 & 0.67 & $(0.49-0.93)$ & & - & - & \\
\hline History of travel & & & & 0.564 & & & - \\
\hline No & 514 & - & - & & - & - & \\
\hline Yes & 155 & 1.13 & $(0.75-1.70)$ & & - & - & \\
\hline Type of participant & & & & $<0.001$ & & & $<0.001$ \\
\hline Patient & 485 & - & - & & - & - & \\
\hline Companion & 184 & 0.27 & $(0.18-0.40)$ & & 0.35 & $(0.23-0.52)$ & \\
\hline Education & & & & 0.103 & & & - \\
\hline None & 385 & - & - & & - & - & \\
\hline Primary & 246 & 1.05 & $(0.74-1.48)$ & & - & - & \\
\hline Secondary & 38 & 0.48 & $(0.24-1.00)$ & & - & - & \\
\hline Bed net use & & & & 0.203 & & & - \\
\hline Yes & 626 & - & - & & - & - & \\
\hline No & 43 & 1.55 & $(0.78-3.08)$ & & - & - & \\
\hline Facility type & & & & 0.300 & & & - \\
\hline Barangay health station & 200 & - & - & & - & - & \\
\hline Rural health unit & 85 & 2.08 & $(0.86-5.03)$ & & - & - & \\
\hline Rapid diagnostic testing center & 384 & 1.21 & $(0.76-1.92)$ & & - & - & \\
\hline
\end{tabular}

${ }^{*}$ Activities include child play, school, and work-related activities.

surveillance and better target interventions. In areas where malaria is no longer endemic, the approach provides valuable information to confirm the absence of malaria in preelimination settings.

By applying this approach to sites with the differing transmission trends in the Philippines, we demonstrate how health facility surveys can complement existing malaria surveillance efforts. In the high-transmission site of Palawan, we identified widespread infections in the community in addition to individuals seeking treatment. Also, with high proportion of PCR positives among companions in these health facilities than others, this emphasizes that these individuals must be tested, especially in facilities that report high numbers of malaria. By screening companions, surveys were extended to wider demographic groups not captured by routine surveillance, particularly older age-groups. Notably, risks of infection did not differ between patients and companions, suggesting equal probabilities of infections between these two groups. This included a substantial proportion of companions who were not seeking treatment but had active febrile illnesses (26/ 1,369). Previous studies have similarly described wider distributions of infections within populations than are captured at health facilities and highlighted the importance of identifying and targeting these infections. ${ }^{13,31,32}$ This study illustrates how screening easy-access groups of health facility attendees can substantially increase the number of infections detected. By applying tablet-based applications to map the distribution of infections, this enables near real-time mapping of infections to better enable targeting of control measures. ${ }^{10}$ The real-time aspect of this data collection is important because this allows the test results and household location to be linked and mapped immediately.

As explored by Fornace et al., ${ }^{30}$ the use of the convenience sampling of health facility attendees markedly increased 
detection probabilities and spatial coverage of surveillance, particularly in rural populations living in forested areas. Overall, a much wider spatial distribution of infected households was only detected by enhanced surveillance methods. Although we detected higher numbers of infections during the sampling period, this did not reflect the temporal changes of malaria throughout the year. We demonstrated the utility of this method to increase the number of infections detected, but further longitudinal sampling would be required to assess finescale changes over time.

In addition, we demonstrated how health facility data can be used to identify risk factors for malaria infection. Analysis of data from Palawan found risk factors for malaria infection, consistent with other studies within this region, identifying higher risks in male individuals ${ }^{33-36}$ and indigenous populations ${ }^{37-40}$ and individuals not using bed nets. ${ }^{41-43} \mathrm{Al}-$ though no associations were found between occupation and malaria risks, these risk factors may be partially attributed to livelihood activities such as swidden farming, movements into forested areas, and associated travel and overnight stays at outdoor locations. ${ }^{44-46}$ Because we also included molecular diagnostics in this approach, we identified significant numbers of subpatent infections, particularly in older age-groups. This is consistent with other studies observing decrease in the risk of patent infections with age, suggestive of acquired immunity. ${ }^{47-49}$ High proportions of subpatent malaria infections may contribute substantially to the transmission and undermine malaria elimination efforts. ${ }^{50}$ This study illustrates how health facility surveys can be used to identify and target these infections. Because this methodology collected geolocated data on the use of bed nets and other preventive measures as well as infection risks, this could be used to identify priority areas for targeting control measures.

As well as identifying infections, this survey methodology allows for verification of the absence of malaria transmission. Two of the study sites, Occidental Mindoro and Bataan, recorded no active infections. This is consistent with public health data and supports the notion that malaria transmission is all but absent in these areas. Although routinely collected surveillance data are key to $\mathrm{WHO}$ certification, augmenting these data with periodic pulses of enhanced passive or active detection provides additional assurance for the absence of infection. ${ }^{51}$ This can improve the statistical robustness of any assertions, especially if conducted at times when historically the transmission would have been high. The use of enhanced surveys might also allow for certification of elimination at lower administrative levels and assist in the more rational use of public health resources.

Despite the utility of this survey methodology, there were several important limitations to this study. This analysis relied on individuals reporting to participating health facilities and therefore is not representative of the wider population within this region. Previous studies have found biases in the demographic groups captured by facility surveys, with high attendance primarily by mothers and young children. ${ }^{52}$ Moreover, the indigenous populations are known to be mobile and may attend different facilities, affecting the relevance of geolocation data for follow-up activities. Because these movements are seasonal, future studies could explore targeting specific time periods. In addition, although most of the infections on Palawan were by $P$. falciparum, approximately a quarter was due to $P$. vivax; this may lead to overestimation of numbers of malaria infections if repeated reports are due to relapses. We also observed individuals $(1.1 \%, n=61)$ who were microscopy- and/or RDT-positive but PCR-negative. With this, there is the possibility of false-positive RDT results when the malaria parasite is cleared, and parasite antigens remain in circulation. These negative results by PCR could result from improper collection and/or storage of DBSs from the study sites to RITM laboratories in Manila, leading to DNA degradation. ${ }^{53-55}$

Nevertheless, this study demonstrates the utility of health facility surveys. Similar health facility-based approaches have been applied in Kenya, ${ }^{56}$ showing good concordance between the facility and community-based estimates of infection. The approach has been used to identify risk factors for infection in both $\mathrm{Haiti}^{57}$ and Indonesia. ${ }^{10}$ In this study, the addition of the combination of geolocation and diagnostic methods performed by community volunteer health workers allowed real-time mapping of field diagnostic methods such as microscopy and RDT down to the household level. This is encouraging as it suggests that as strategies emerge for malaria elimination, these health workers can take new roles with proper training and resources. This is evident as they adapted to the use of mobile technology for tablet-based questionnaires and mapping and collect blood on filter papers.

\section{CONCLUSION}

Extended health facility surveys can provide more comprehensive and readily accessible data for operational planning and evaluation of malaria and other diseases. Incorporating molecular diagnostics provided additional information in detecting subpatent and asymptomatic infections that are missed by routine methods such as microscopy and RDT, preventing underestimated malaria prevalence. How this approach can be incorporated into the routine health system and budgets require further consideration. Community volunteer health workers can collect blood on filter papers for multiple testing or multi-disease testing in the future. Indeed, health facility surveys incorporated with geolocation and molecular methods could be adapted across a range of ecologies (e.g., rural and forested population) and can support malaria control not just in Palawan but other areas with similar transmission. Similarly, these methods can be used to provide stronger evidence of progress toward elimination as observed in Occidental Mindoro and Bataan allowing subnational verification as part of the Philippines' march to malaria freedom.

Received July 7, 2020. Accepted for publication October 15, 2020.

Published online January 18, 2021.

Note: Supplemental tables appear at www.ajtmh.org.

Acknowledgments: We would like to acknowledge the Newton Fund, Philippine Council for Health Research and Development, and U.K. Medical Research Council for funding received for ENSURE: Enhanced surveillance for control and elimination of malaria in the Philippines (MR/N019199/1). Also, we are grateful to Ellaine Hernandez and Carol Joy Sarsadiaz for assisting the fieldwork activities of the project. Also, we thank the local government and health staff of Rizal, Palawan, for supporting the implementation of this survey.

Authors' addresses: Ralph A. Reyes, Maria Lourdes M. Macalinao, Beaulah L. Boncayao, Ellaine S. De La Fuente, Hennessey M. Sabanal, Alison Paolo N. Bareng, Inez Andrea P. Medado, Edelwisa S. Mercado, Jennifer S. Luchavez, and Fe Esperanza J. Espino, Research Institute for Tropical Medicine, Manila, Philippines, E-mails: rreyes. 
rmt@gmail.com, maloumacalinao@gmail.com, beaulah_0719944@ yahoo.com, ellainesdelafuente@gmail.com, hmsabanal@gmail.com, pbareng@yahoo.com, iapmedado.ritm@gmail.com, esegubre.mercado@ gmail.com, jluchavez@yahoo.com, and fe.espino2019@gmail.com. Kimberly M. Fomace, Julius Clemence R. Hafalla, and Chris J. Drakeley, London School of Hygiene and Tropical Medicine, London, United Kingdom, E-mails: kimberly.fornace@|shtm.ac.uk, julius.hafalla@|shtm.ac.uk, and chris. drakeley@ishtm.ac.uk. Mario S. Baquilod, MIMAROPA Center for Health Development, Quezon, Philippines, E-mail: mariobaquilod2@ gmail.com.

This is an open-access article distributed under the terms of the Creative Commons Attribution (CC-BY) License, which permits unrestricted use, distribution, and reproduction in any medium, provided the original author and source are credited.

\section{REFERENCES}

1. DOH - NMCEP, 2018. Department of Health - National Malaria Control and Elimination Program, Philippines, Malaria Manual of Operations. Manila, Philippines: DOH.

2. DOH - Philippines, WHO, UCSF, 2015. Eliminating Malaria: Case-Study 6, Progress towards Subnational Elimination in the Philippines. Geneva, Switzerland: WHO.

3. World Health Organization, 2018. Malaria Surveillance, Monitoring and Evaluation: A Reference Manual. Geneva, Switzerland: WHO.

4. Stevenson JC et al., 2013. Reliability of school surveys in estimating geographic variation in malaria transmission in the western Kenyan highlands. PLoS One 8: e77641.

5. Stresman GH, Stevenson JC, Ngwu N, Marube E, Owaga C, Drakeley C, Bousema T, Cox J, 2014. High levels of asymptomatic and subpatent Plasmodium falciparum parasite carriage at health facilities in an area of heterogeneous malaria transmission intensity in the Kenyan highlands. Am J Trop Med Hyg 91: 1101-1108.

6. Okebe J, Affara M, Correa S, Muhammad AK, Nwakanma D, Drakeley C, D'Alessandro U, 2014. School-based countrywide seroprevalence survey reveals spatial heterogeneity in malaria transmission in the Gambia. PLoS One 9: e110926.

7. Tin SS, Wiwanitkit V, 2014. Asymptomatic malaria in apparently healthy schoolchildren. J Vector Borne Dis 51: 349.

8. Ashton RA et al., 2015. Geostatistical modeling of malaria endemicity using serological indicators of exposure collected through school surveys. Am J Trop Med Hyg 93: 168-177.

9. Bousema T, Griffin JT, Sauerwein RW, Smith DL, Churcher TS, Takken W, Ghani A, Drakeley C, Gosling R, 2012. Hitting hotspots: spatial targeting of malaria for control and elimination. PLoS Med 9: e1001165

10. Fornace $\mathrm{KM}$ et al., 2018. Use of mobile technology-based participatory mapping approaches to geolocate health facility attendees for disease surveillance in low resource settings. Int $J$ Health Geogr 17: 21.

11. Björkman $A B, 2018$. Asymptomatic low-density malaria infections: a parasite survival strategy? Lancet Infect Dis 18: 485-486.

12. Chourasia MK et al., 2017. Additional burden of asymptomatic and sub-patent malaria infections during low transmission season in forested tribal villages in Chhattisgarh, India. Malar $J$ 16: 320 .

13. Zhou G, Afrane YA, Malla S, Githeko AK, Yan G, 2015. Active case surveillance, passive case surveillance and asymptomatic malaria parasite screening illustrate different age distribution, spatial clustering and seasonality in western Kenya. Malar J 14: 41.

14. Okell LC, Bousema T, Griffin JT, Ouedraogo AL, Ghani AC, Drakeley CJ, 2012. Factors determining the occurrence of submicroscopic malaria infections and their relevance for control. Nat Commun 3: 1237.

15. Okell LC, Ghani AC, Lyons E, Drakeley CJ, 2009. Submicroscopic infection in Plasmodium falciparum-endemic populations: a systematic review and meta-analysis. J Infect Dis 200: 1509-1517.

16. Wu L, van den Hoogen LL, Slater H, Walker PG, Ghani AC, Drakeley CJ, Okell LC, 2015. Comparison of diagnostics for the detection of asymptomatic Plasmodium falciparum infections to inform control and elimination strategies. Nature 528: S86-S93.
17. Grignard L, Shah S, Chua T, William T, Drakeley C, Fornace K, 2019. Natural human infections with Plasmodium cynomolgi and other malaria species in an elimination setting in Sabah, Malaysia. J Infect Dis 220: 1946-1949.

18. Wen S et al., 2016. Targeting populations at higher risk for malaria: a survey of national malaria elimination programmes in the Asia Pacific. Malar J 15: 271.

19. Department of Health, 2019. Thematic Desk Review 2019. National Malaria Control and Elimination Program. Manila, Philippines: DOH.

20. Philippine Statistics Authority, 2003. 2000 Census of Population and Housing (Report No. 2, Vol. 1 - Demographic and Housing Characteristics): Palawan. Available at: https://psa.gov.ph/sites/ default/files/Palawan.pdf. Accessed June 12, 2020.

21. Philippine Statistics Authority, 2003. 2000 Census of Population and Housing (Report No. 2, Vol. 1 - Demographic and Housing Characteristics): Occidental Mindoro. Available at: https://psa. gov.ph/sites/default/files/Occ\%20Mindoro.pdf. Accessed June 12, 2020.

22. Philippine Statistics Authority, 2003. 2000 Census of Population and Housing (Report No. 2, Vol. 1 - Demographic and Housing Characteristics): Bataan. Available at: https://psa.gov.ph/sites/ default/files/bataan_0.pdf. Accessed June 12, 2020.

23. DNA Analyst Training-Laboratory Training Manual, 2006. Protocol 3.05 Chelex® 100 Non-differential Extraction. Largo, FL: National Forensic Science Technology Center.

24. Lee KS, Divis PC, Zakaria SK, Matusop A, Julin RA, Conway DJ, Cox-Singh J, Singh B, 2011. Plasmodium knowlesi: reservoir hosts and tracking the emergence in humans and macaques. PLoS Pathog 7: e1002015.

25. Snounou G, Singh B, 2002. Nested PCR analysis of Plasmodium parasites. Methods Mol Med 72: 189-203.

26. Snounou G, Viriyakosol S, Jarra W, Thaithong S, Brown KN, 1993. Identification of the four human malaria parasite species in field samples by the polymerase chain reaction and detection of a high prevalence of mixed infections. Mol Biochem Parasit 58: 283-292.

27. Calderaro A, Piccolo G, Perandin F, Gorrini C, Peruzzi S, Zuelli C, Snounou G, 2007. Genetic polymorphisms influence Plasmodium ovale PCR detection accuracy. J Clin Microbiol 45: 1624-1627.

28. QGIS.org, 2019. QGIS Geographic Information System. Open Source Geospatial Foundation Project. Available at: http:// qgis.org.

29. R Core Team, 2020. R: A Language and Environment for Statistical Computing. Vienna, Austria: R Foundation for Statistical Computing. Available at: http://www.R-project.org/.

30. Fornace K, Reyes R, Macalinao M, Bareng A, Luchavez J, Hafalla $J$, Espino F, Drakeley C, 2020. Disentangling fine-scale effects of environment on malaria detection and infection to design risk-based disease surveillance systems in changing landscapes. Medrxiv. doi:10.1101/2020.04.15.20065656.

31. Oduro AR, Maya ET, Akazili J, Baiden F, Koram K, Bojang K, 2016. Monitoring malaria using health facility based surveys: challenges and limitations. BMC Public Health 16: 354.

32. Sesay SSS, Giorgi E, Diggle PJ, Schellenberg D, Lalloo DG, Terlouw DJ, 2017. Surveillance in easy to access population subgroups as a tool for evaluating malaria control progress: a systematic review. PLoS One 12: e0183330.

33. Lansang MAD, Belizario VY, Bustos MDG, Saul A, Aguirre A, 1997. Risk factors for infection with malaria in a low endemic community in Bataan, the Philippines. Acta Trop 63: 257-265.

34. Ramdzan AR, Ismail A, Mohd Zanib ZS, 2020. Prevalence of malaria and its risk factors in Sabah, Malaysia. Int $J$ Infect Dis 91: 68-72.

35. Smith JL, Auala J, Haindongo E, Uusiku P, Gosling R, Kleinschmidt I, Mumbengegwi D, Sturrock HJ, 2017. Malaria risk in young male travellers but local transmission persists: a case-control study in low transmission Namibia. Malar J 16: 70.

36. Tesfahunegn A, Berhe G, Gebregziabher E, 2019. Risk factors associated with malaria outbreak in Laelay Adyabo district northern Ethiopia, 2017: case-control study design. BMC Public Health 19: 484.

37. Matsumoto-Takahashi ELA, Tongol-Rivera P, Villacorte EA, Angluben RU, Jimba M, Kano S, 2018. Bottom-up approach to 
strengthen community-based malaria control strategy from community health workers' perceptions of their past, present, and future: a qualitative study in Palawan, Philippines. Trop Med Health 46: 24.

38. Matsumoto-Takahashi ELA, Kano S, 2016. Evaluating active roles of community health workers in accelerating universal access to health services for malaria in Palawan, the Philippines. Trop Med Health 44: 10.

39. Matsumoto-Takahashi ELA, Tongol-Rivera P, Villacorte EA, Angluben RU, Jimba M, Kano S, 2015. Patient knowledge on malaria symptoms is a key to promoting universal access of patients to effective malaria treatment in palawan, the Philippines. PLoS One 10: e0127858.

40. Sundararajan R, Kalkonde Y, Gokhale C, Greenough PG, Bang A, 2013. Barriers to malaria control among marginalized tribal communities: a qualitative study. PLoS One 8: e81966.

41. Fokam EB, Dzi KTJ, Ngimuh L, Enyong P, 2016. The effect of long lasting insecticide bed net use on malaria prevalence in the Tombel health district, south west region-Cameroon. MalarRes Treat 2016: 3216017.

42. Levitz L, Janko M, Mwandagalinwa K, Thwai KL, Likwela JL, Tshefu AK, Emch M, Meshnick SR, 2018. Effect of individual and community-level bed net usage on malaria prevalence among under-fives in the Democratic Republic of Congo. Malar J 17: 39.

43. Liu H, Xu J, Guo X, Havumaki J, Lin Y, Yu G, Zhou D, 2015. Coverage, use and maintenance of bed nets and related influence factors in Kachin Special Region II, northeastern Myanmar. Malar J 14: 212.

44. Bannister-Tyrrell $M$ et al., 2019. Forest goers and multidrugresistant malaria in Cambodia: an ethnographic study. $A m \mathrm{~J}$ Trop Med Hyg 100: 1170-1178.

45. Kar NP, Kumar A, Singh OP, Carlton JM, Nanda N, 2014. A review of malaria transmission dynamics in forest ecosystems. Parasit Vectors 7: 265.

46. Nath DC, Mwchahary DD, 2012. Malaria prevalence in forest and nonforest areas of Kokrajhar district of Assam. Internat Scholarly Res Not 7: 265.

47. Griffin JT, Ferguson NM, Ghani AC, 2014. Estimates of the changing age-burden of Plasmodium falciparum malaria disease in sub-Saharan Africa. Nat Commun 5: 3136.
48. Millar J, Psychas P, Abuaku B, Ahorlu C, Amratia P, Koram K, Oppong S, Valle D, 2018. Detecting local risk factors for residual malaria in northern Ghana using Bayesian model averaging. Malar J 17: 343.

49. Protopopoff N, Van Bortel W, Speybroeck N, Van Geertruyden JP, Baza D, D'Alessandro U, Coosemans M, 2009. Ranking malaria risk factors to guide malaria control efforts in African highlands. PLoS One 4: e8022.

50. Slater HC et al., 2019. The temporal dynamics and infectiousness of subpatent Plasmodium falciparum infections in relation to parasite density. Nat Commun 10: 1433.

51. Stresman G, Cameron A, Drakeley C, 2017. Freedom from infection: confirming interruption of malaria transmission. Trends Parasitol 33: 345-352.

52. Surendra $\mathrm{H}$ et al., 2020. Using health facility-based serological surveillance to predict receptive areas at risk of malaria outbreaks in elimination areas. BMC Med 18: 9.

53. Zainabadi K, Adams M, Han ZY, Lwin HW, Han KT, Ouattara A, Thura S, Plowe CV, Nyunt MM, 2017. A novel method for extracting nucleic acids from dried blood spots for ultrasensitive detection of low-density Plasmodium falciparum and Plasmodium vivax infections. Malar J 16: 377.

54. Zainabadi K, Nyunt M, Plowe C, 2019. An improved nucleic acid extraction method from dried blood spots for amplification of Plasmodium falciparum kelch13 for detection of artemisinin resistance. Malar J 18: 192.

55. Hwang $\mathrm{J}$ et al., 2012. Long-term storage limits PCR-based analyses of malaria parasites in archival dried blood spots. Malar $J$ 11: 339.

56. Stresman GH, Stevenson JC, Ngwu N, Marube E, Owaga C, Drakeley C, Bousema T, Cox J, 2014. High levels of asymptomatic and subpatent Plasmodium falciparum parasite carriage at health facilities in an area of heterogeneous malaria transmission intensity in the Kenyan highlands. Am J Trop Med Hyg 91: 1101-1108.

57. Ashton RA et al., 2020. Risk factors for malaria infection and seropositivity in the elimination area of Grand'Anse, Haiti: a case-control study among febrile individuals seeking treatment at public health facilities. Am J Trop Med Hyg 103: 767-777. 